\title{
Morningside Medals, the Chern Prizes, and the International Cooperation Awards
}

\author{
excerpts from the 2010 ICCM booklet
}

\section{Morningside medals of mathematics and applied mathematics}

The Morningside Medal of Mathematics is awarded to outstanding mathematicians of Chinese descent to encourage them in their pursuit of mathematical truth. Up to six medals, two gold and four silver, are awarded every three years at the International Congress of Chinese
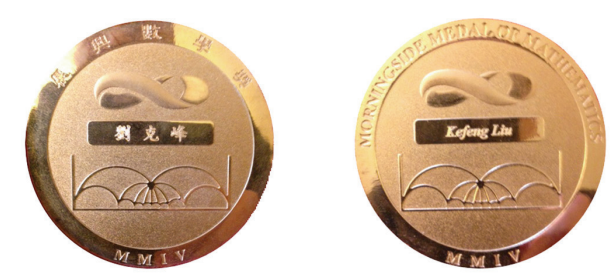

The Morningside Gold Medal awarded to Kefeng Liu in 2004

Mathematicians. The Morningside Medals also carry a cash award of US \$25,000 for each of the gold medalists; and US \$ 10,000 for each of the silver medalists. A committee of internationally renowned mathematicians, chaired by Professor Shing-Tung Yau, selects the medalists.

In addition to the name of medalists, each gold and silver medal has the image of a Möbius band and a fundamental domain. They were chosen not only for the simplicity of the image, but also because of their significant contributions to the development of mathematics in 20th century.

The Möbius band (also known as the Möbius strip) was discovered in 1858 by the German mathematician and astronomer August Ferdinand Möbius. This curious one-sided surface does not have any orientation, yet has a distinct topographical character. It was one of the most important discoveries of the 20th century, which has profoundly influenced modern physics, classical physics as well as modern mathematics, including geometry.
A fundamental domain is related to the concept of a group, which can be used to express symmetry in mathematics. During the late 19th century, infinite discrete group was studied but only until the 20th century, did it become a main subject area in mathematics. This field of study is not only important in geometry but also in number theory. Practically all the famous developments in modern number theory are related to concepts of fundamental domain and discrete group.

\section{Morningside Medals of Mathematics Selection Committee}

The selection committee for the Morningside Medal of Mathematics is chaired by Professor Shing-Tung Yau. A

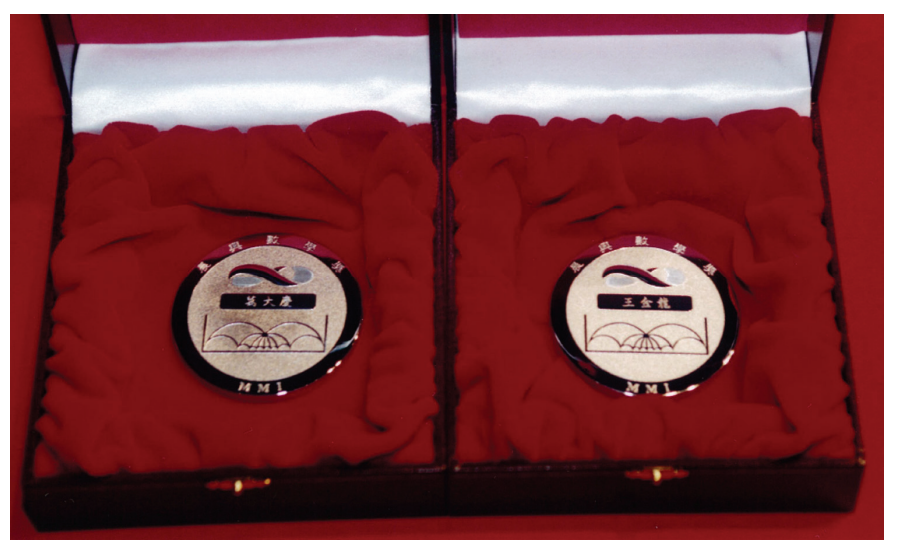

The Morningside Silver Medals awarded to Daqing Wan and Chin-Lung Wang in 2001

nomination committee for the Morningside Medal of Mathematics, comprising a maximum of fifty Chinese mathematicians worldwide, nominates individuals based on their research, qualifications, and vita. Subsequently, the nomination committee submits the names of the nominated individuals, along with supporting materials, to the selection committee. After a thorough multi-step review, the selection committee, comprising leading mathematicians with different research interest, makes a final decision. All the members of the selection commit- 
tee, with the exception of the committee chair, are non-Chinese, thereby ensuring the independence and integrity of their decision.

Some new awards were presented besides the Morningside medals: the Chern Prizes (from ICCM 2001), and the International Cooperation Awards (from ICCM 2004).

\section{The Chern Prize}

The Chern Prize in mathematics was established in 2001 in honor of Professor Shing-Shen Chern, one of the greatest geometers and Chinese mathematicians of the twentieth century. The Chern Prize is presented every three years to mathematicians of Chinese descent who have made exceptional contributions to mathematical research or to public service activities in support of mathematics.

\section{The ICCM International Cooperation Award}

The ICCM International Cooperation Award is presented to an individual who has promoted the development of mathematics in China, Hong Kong, and Taiwan through collaboration, teaching, and support of Chinese mathematicians. The inaugural award was presented at the Third International Congress of Chinese Mathematicians in 2004. The Selection Committee for the ICCM International Cooperation Award in 2004 was chaired by

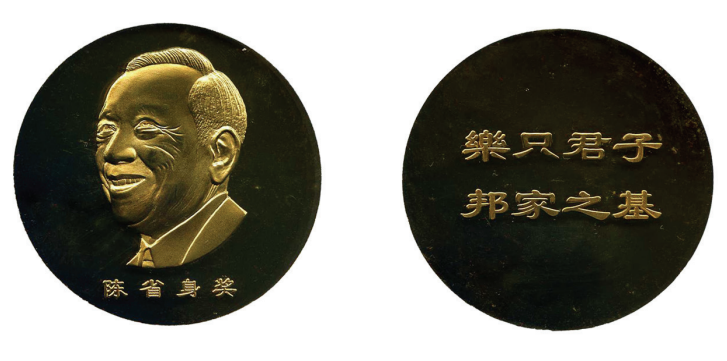

The Chern Prize

Professor Shing-Tung Yau, President of the International Congress of Chinese Mathematicians. The other members of the Selection Committee are Professor Shiu-Yuen Cheng of The Hong Kong University of Science and Technology, Professor Jun Li of Stanford University, Professor Chang-Shou Lin of Taiwan University, Professor Kefeng Liu of the University of California at Los Angeles and Zhejiang University, Professor Jie Xiao of Tsinghua University, Professor Zhouping Xin of The Chinese University of Hong Kong, and Professor Lo Yang of the Chinese Academy of Sciences.

\section{Morningside Gold Medal of Mathematics (Beijing)}

\section{Chang-Shou Lin}

Professor Chang-Shou Lin was awarded the 1998 Morningside Gold Medal of Mathematics for his research work on locating isometric embedding problems, scalar curvature equations and semi-linear elliptic equations. He is now a professor of Mathematics at National Taiwan University.
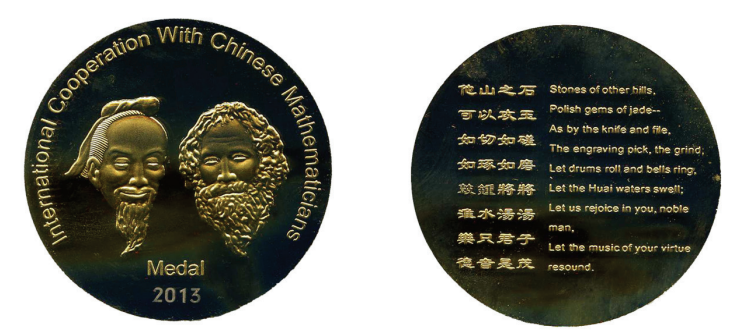

The International Cooperation Award

\section{Shouwu Zhang}

Professor Zhang was awarded the 1998 Morningside Gold Medal of Mathematics for his contributions to number theory and arithmetical algebraic geometry. His achievements include the connexions between $L$-function and arithmetic via the Gross-Zagier formula, the Galois distributions of points on abelian varieties, and pure Arakelov geometry. He is now a professor of Mathematics at Princeton University.

\section{Morningside Silver Medal of Mathematics (Beijing)}

\section{Raymond Hon-fu Chan}

Professor Chan was awarded the 1998 Morningside Silver Medal of Mathematics for his work in computational mathematics. He is a professor of Mathematics at the Chinese University of Hong Kong.

\section{Chong-Qing Cheng}

Professor Cheng was awarded the 1998 Morningside Silver Medal of Mathematics for his contributions in the areas of dynamic systems and Hamiltonian dynamics. He is recognized for his work on problems related to Kolomogorov-Arnold-Moser (KAMtheory). He is a Vice-President and a Cheung Kong Professor of Mathematics at Nanjing University. 


\section{Kefeng Liu}

Professor Liu was awarded the 1998 Morningside Silver Medal of Mathematics for his achievements in topology, geometry, and mathematical physics. He is a professor of Mathematics at University of California at Los Angeles, and a Guangbiao Chair Professor of Mathematics at Zhejiang University.

\section{Tong Yang}

Professor Yang was awarded the 1998 Morningside Silver Medal of Mathematics for his contributions to the fundamental well-posedness problem for general systems of hyperbolic conservation laws. He is a Chair Professor of Mathematics and an Associate Dean of College of Sciences and Engineering at City University of Hong Kong.

\section{Morningside Gold Medal of Mathematics (Taipei)}

\section{Jun $L i$}

Professor Li was awarded the 2001 Morningside Gold Medal of Mathematics for his fundamental contributions to the study of moduli spaces of vector bundles and to the theory of stable maps and basic invariants of Calabi-Yau manifolds. He is a professor of Mathematics at Stanford University.

\section{Horng-Tzer Yau}

Professor Yau was awarded the 2001 Morningside Gold Medal of Mathematics for his profound contributions to mathematical physics. His achievements are products of his unusual ability to bring physical insights to bear on mathematical problems. He is a professor of Mathematics at Harvard University.

\section{Morningside Silver Medal of Mathematics (Taipei)}

\section{Daqing Wan}

Professor Wan was awarded the 2001 Morningside Silver Medal of Mathematics for his proof of Dwork's conjecture on $L$-functions over finite fields. He is a professor of Mathematics at the University of California at Irvine.

\section{Chin-Lung Wang}

Professor Wang was awarded the 2001 Morningside Silver Medal of Mathematics for his contributions on the birational model of algebraic varieties with singularities. He is a professor of Mathematics at Central University of Taiwan and professor of Mathematics at National Taiwan University.

\section{Sijue $W u$}

Professor Wu was awarded the 2001 Morningside Silver Medal of Mathematics for her establishment of local well-posedness of the water wave problems in a Sobolev class in arbitrary space dimensions. He is a professor of Mathematics at University of Michigan at Ann Arbor.

\section{Nanhua Xi}

Professor Xi was awarded the 2001 Morningside Silver Medal of Mathematics for his work on solving an important conjecture of Lusztig. He is a professor of the

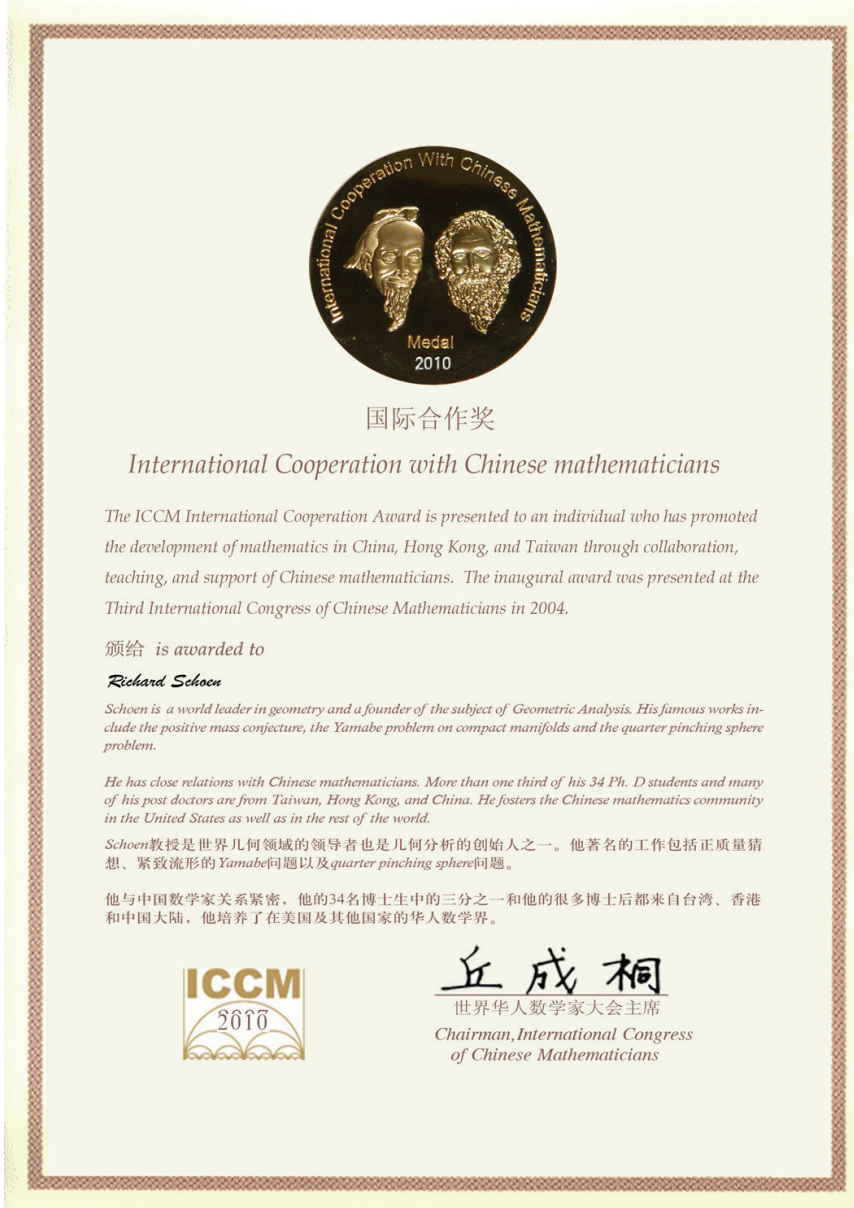

The International Cooperation Award was presented to Richard Schoen in 2010

Academy of Mathematics and Systems Sciences in Chinese Academy of Sciences.

\section{Chern Prize}

\section{Song-Sun Lin}

Professor Lin was awarded the 2001 Chern Prize for his distinguished contributions to the development of nonlinear partial differential equations and dynamical 
systems. He is a Chair Professor of Applied Mathematics at Chiao-Tung University, Taiwan.

\section{Jiu-Kang $\mathrm{Yu}$}

Professor Yu was awarded the 2001 Chern Prize for his important contributions to number theory, algebraic geometry, and representation theory. He is a professor of Mathematics at Purdue University.

\section{Morningside Gold Medal of Mathematics (Hong Kong)}

\section{Kefeng Liu}

Professor Liu was awarded the 2004 Morningside Gold Medal of Mathematics for his major achievements in the research fields of Witten's rigidity theorem and elliptic genus, mathematical theory of Mirror principle, and moduli spaces in algebraic geometry. He is a professor of Mathematics at the University of California at Los Angeles and the Guangbiao Chair Professor of Mathematics at Zhejiang University.

\section{Zhouping Xin}

Professor Xin was awarded the 2004 Morningside Gold Medal of Mathematics for his research in nonlinear partial differential equations. In the area of boundary layer theory, he proved the global existence of solutions of the Prandtl equations. He also established a new mathematical framework for the study of transonic shockwave flow in a nozzle, and within this framework, he addressed and confirmed an old conjecture of Courant and Friedrichs by establishing the stability of such shocks. He is the William M.W. Mong Professor of Mathematics at The Chinese University of Hong Kong.

\section{Morningside Gold Medal of Applied Mathematics (Hong Kong)}

\section{Thomas Yizhao Hou}

Professor Hou was awarded the 2004 Morningside Gold Medal of Applied Mathematics for his seminal research on applied partial differential equations, scientific computation and numerical analysis. This research includes convergence of the point vortex method, accurate numerical methods for fluid interfaces with surface tension, analysis of three dimensional vortex sheets, and singularity criteria for the three dimensional Euler equation. He is the Charles Lee Powell Professor of Applied Mathematics and Computational Mathematics at the California Institute of Technology.

\section{Zhiliang Ying}

Professor Ying was awarded the 2004 Morningside Gold Medal of Applied Mathematics for his fundamental contributions and breakthroughs in several areas of mathematical statistics and stochastic processes, including survival analysis, counting processes, adaptive design, and their applications to other fields such as cancer and AIDS research, applied psychological measurement, and econometrics. He is a professor of Statistics at Columbia University.

\section{Morningside Silver Medal of Mathematics (Hong Kong)}

\section{Jin-Yi Cai}

Professor Cai was awarded the 2004 Morningside Silver Medal of Mathematics for his contributions in the resolution of two long-standing open conjectures by Juris Hartmanis on sparse sets, and other important discoveries in computational complexity. He is a professor of Computer Science and adjunct professor of Mathematics at the University of Wisconsin at Madison.

\section{Ai-Ko Liu}

Professor Liu was awarded the 2004 Morningside Silver Medal of Mathematics for his fundamental contributions to both the Seiberg-Witten theory and the topology of symplectic 4-manifolds. He has developed the family Seiberg-Witten theory and applied it with spectacular success to the enumerations of modal curves in algebraic surfaces. He was an assistant professor of Mathematics at the University of California at Berkeley.

\section{Xi-Ping Zhu}

Professor Zhu was awarded the 2004 Morningside Silver Medal of Mathematics for his contributions to the Ricci flow on Kähler manifolds, and the study of geometry of complete Kähler manifolds of positive curvature. He is a professor of Mathematics at Zhongshan University and a Senior Professor at the Center of Mathematical Sciences at Zhejiang University.

\section{Chern Prize}

\section{Lo Yang}

Professor Yang was awarded the 2004 Chern Prize for his dedication to the development of mathematics in China and for his role in promoting mathematical activities and endeavors over the past twenty years. He is the Founding Director of the Academy of Mathematics and Systems Sciences and Deputy Director of the Morningside Center of Mathematics. 


\section{Fanghua Lin}

Professor Fang was awarded the 2004 Chern Prize for his work on the theory of liquid crystals, harmonic maps, Ginzburg-Landau equations, the static as well as dynamic theory of topological defects, Skyrme and Faddeev models, and the Navier-Stokes equations. He is a professor of Mathematics at New York University.

\section{ICCM International Cooperation Award}

\section{John H. Coates}

The inaugural ICCM International Cooperation Award was given to Professor John Coates for his dedication and devotion to nurturing Chinese mathematicians over the past twenty years. Professor Coates has trained many Chinese students and post-doctoral fellows, and he has been a tremendous influence on their growth as mathematicians. He has selflessly given his time and energy to helping young Chinese mathematicians in secondary schools as well as those engaged in advanced research. Professor Coates embodies the qualities revered by the mathematicians who have benefited from his tutelage: intellectual rigor, respect for others and learning, kindness, and the ability to transcend language and culture. He is the Sadleirian Professor of Pure Mathematics.

\section{Morningside Gold Medal of Mathematics (Hangzhou)}

\section{Xu-Jia Wang}

Professor Wang is awarded the 2007 Morningside Gold Medal of Mathematics for his outstanding contributions to the theory of fully nonlinear elliptic equations, Hessian measures and their applications, and for his remarkable solutions to a set of long-standing open problems in affine and differential geometry. Professor Wang is a professor at the Centre for Mathematics and its Applications at Australian National University.

\section{Morningside Gold Medal of Applied Mathematics (Hangzhou)}

\section{Jianqing Fan}

Professor Fan is awarded the 2007 Morningside Gold Medal of Applied Mathematics for his seminal work in nonparametric modeling and inferences, for his fundamental contributions to high-dimensional statistical learning, nonlinear time series and bio-statistics, and his achievements toward the development of novel statistical techniques in finance and molecular biology. Professor Fan is the Frederick L. Moore Professor of Finance and a professor of Statistics at Princeton University, where he also serves as the Director of the Committee for Statistical Studies.

\section{Morningside Silver Medal of Mathematics (Hangzhou)}

\section{Сhiu-Chu Liu}

Professor Liu is awarded the 2007 Morningside Silver Medal of Mathematics for her significant contributions to the definition of open Gromov-Witten invariants, to the mathematical theory of topological vertex, and to the new definition of quasi-local mass in general relativity. Professor Liu is an associate professor at Columbia University.

\section{Lizhen Ji}

Professor Lizhen Ji is awarded the 2007 Morningside Silver Medal of Mathematics for his important work on

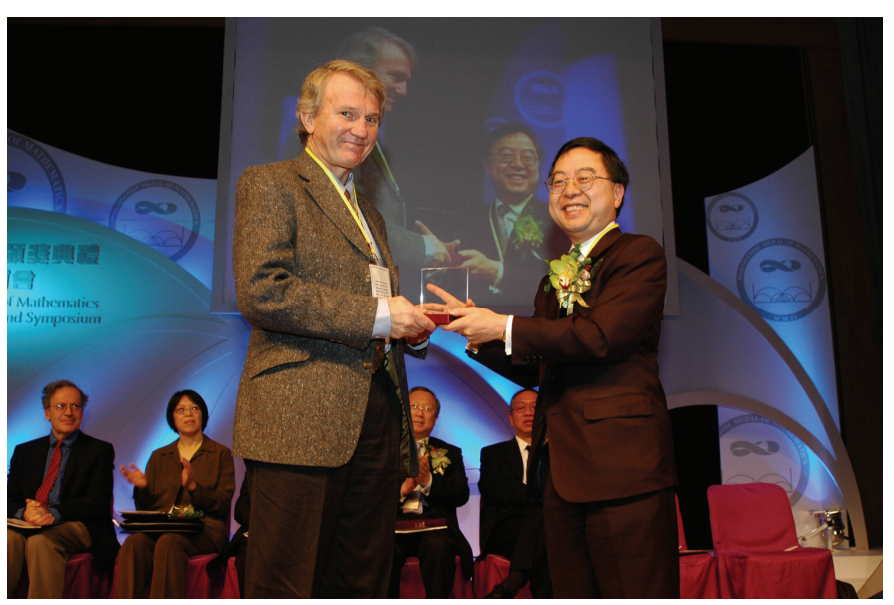

Left: John Coates, the 2004 International Cooperation Awardee

spectral geometry, for his proof of the trace class conjecture in the theory of automorphic forms, and for his study of various compactifications of symmetric and locally symmetric spaces. Professor Ji is a professor of Mathematics at the University of Michigan at Ann Arbor.

Shi Jin

Professor Jin is awarded the 2007 Morningside Silver Medal of Mathematics for his significant research on the development of computational methods for high frequency and quantum waves in heterogeneous media and for multi-scale methods for kinetic and hyperbolic equations. Professor Jin is a professor of Mathematics at the University of Wisconsin at Madison.

\section{Chiun-Chuan Chen}

Professor Chen is awarded the 2007 Morningside Silver Medal of Mathematics for his significant research contribution to nonlinear elliptic equations, and to the 
regularity of Navier-Stokes equations. He is a professor of Mathematics at the National Taiwan University.

\section{Ye Tian}

Professor Tian is awarded the 2007 Morningside Sil-
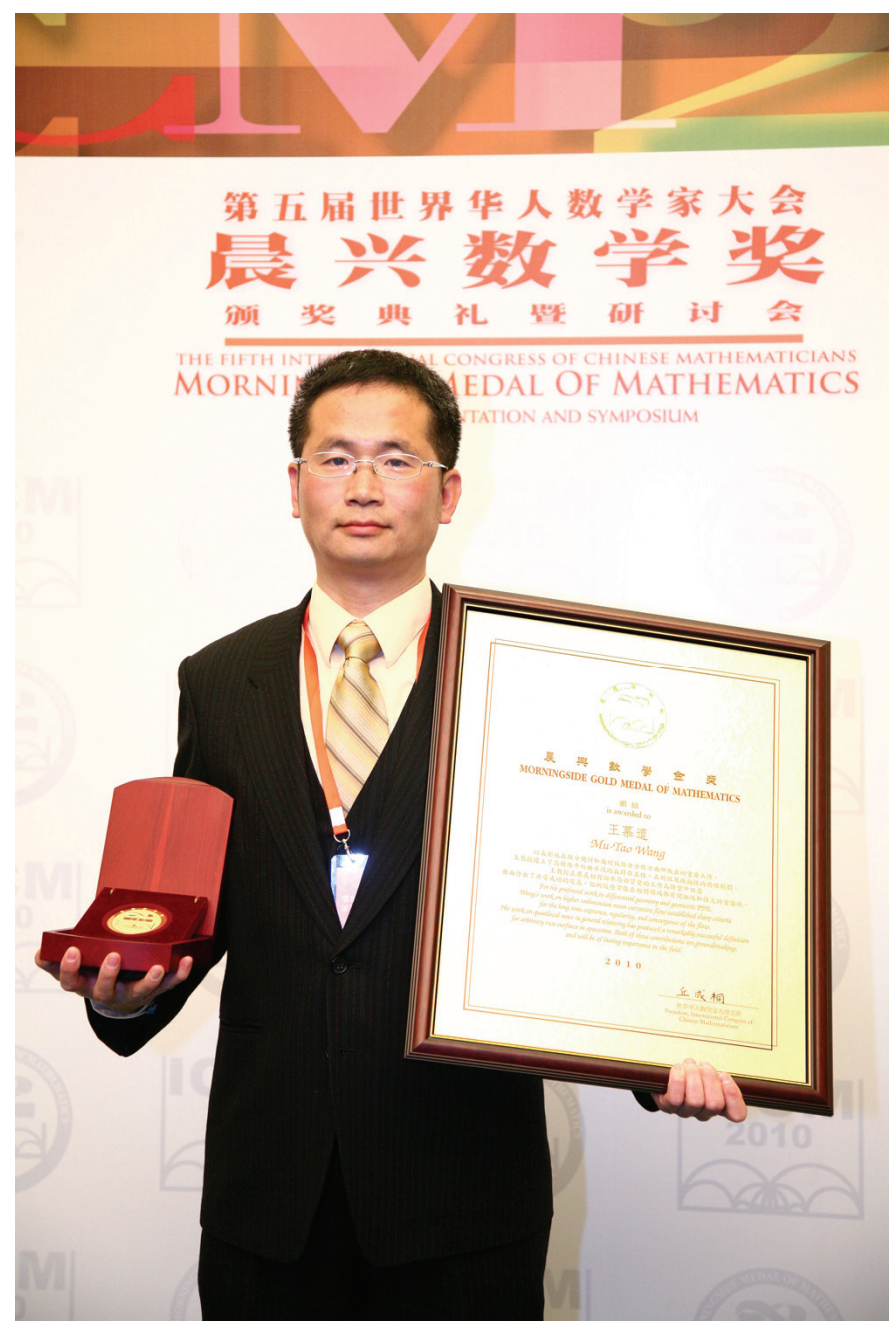

2010 ICCM Gold Medalist, Mu-Tao Wang

ver Medal of Mathematics for his important work on diophantine equations via the study of the conjecture of Birch and Swinnerton-Dyer, for both elliptic curves and certain abelian varieties with complex multiplication. Professor Tian holds a joint appointment as professor of Mathematics at the Academy of Mathematics and Systems Science and at the Morningside Center of Mathematics of the Chinese Academy of Sciences.

\section{Chern Prize}

\section{Mu-Tao Wang}

Professor Wang is awarded the 2007 Chern Prize for his research on geometric partial differential equations and general relativity, including higher codimensional mean curvature flow, problems related to higher codimensional minimal submanifold and superrigidity theorem for representations of certain discrete groups. Professor Wang is currently a professor of Mathematics at Columbia University.

\section{Shiu-Yuen Cheng}

Professor Cheng is awarded the 2007 Chern Prize for his fundamental contributions to the geometric study of eigenfunctions and eigenvalues of Laplacian, which has extensive application in geometric understanding of manifolds. In addition to being a leader in geometric analysis, he also taught and mentored numerous mathematicians in China. Many of these students went to the United States for further study and subsequently became leaders in their field. Professor Cheng is currently Dean of Science at The Hong Kong University of Science and Technology.

\section{ICCM International Cooperation Award}

\section{Stanley J. Osher}

Professor Osher is awarded the second ICCM International Cooperation Award for his tremendous contributions to the training of Chinese applied mathematicians, for his help and participation in many scientific conferences and workshops in China and Hong Kong, and for his significant research achievements in computational mathematics. Professor Osher is a professor of Mathematics at University of California at Los Angeles.

\section{Morningside Gold Medal of Mathematics (Beijing)}

\section{Mu-Tao Wang}

Professor Wang is awarded the 2010 Morningside Gold Medal of Mathematics for his profound work in differential geometry and geometric PDE. Wang's work on higher codimension mean curvature flow established sharp criteria for the long time existence, regularity, and convergence of the flow. His work on quasilocal mass in general relativity has produced a remarkably successful definition for arbitrary two surfaces in spacetime. Both of these contributions are groundbreaking, and will be of lasting importance in the field. Professor Wang is a Professor of Mathematics at Columbia University. His research interests include differential geometry, discrete groups, partial differential equations and general relativity. He received his B.S. and M.S. in mathematics from Taiwan University and his Ph.D. in mathematics from Harvard University. Prior to joining Columbia University, Professor Wang has taught at Stanford University. Among his awards and honors are the Chern Prize awarded at 
ICCM 2007, a Kavli Fellowship in 2007, a Sloan Research Fellowship, for 2003-2005, the Harold M. Bacon Memorial Teaching Award from Stanford University in 2000, and a U.S. National Science Foundation Grant since 2001.

\section{Sijue $W u$}

Professor Wu is awarded the 2010 Morningside Gold Medal of Mathematics for her outstanding contributions to the global well-posedness of small amplitude solution to the water wave problems in both 2-D and 3-D. Professor Wu is the Robert W. and Lynne H. Browne Professor of

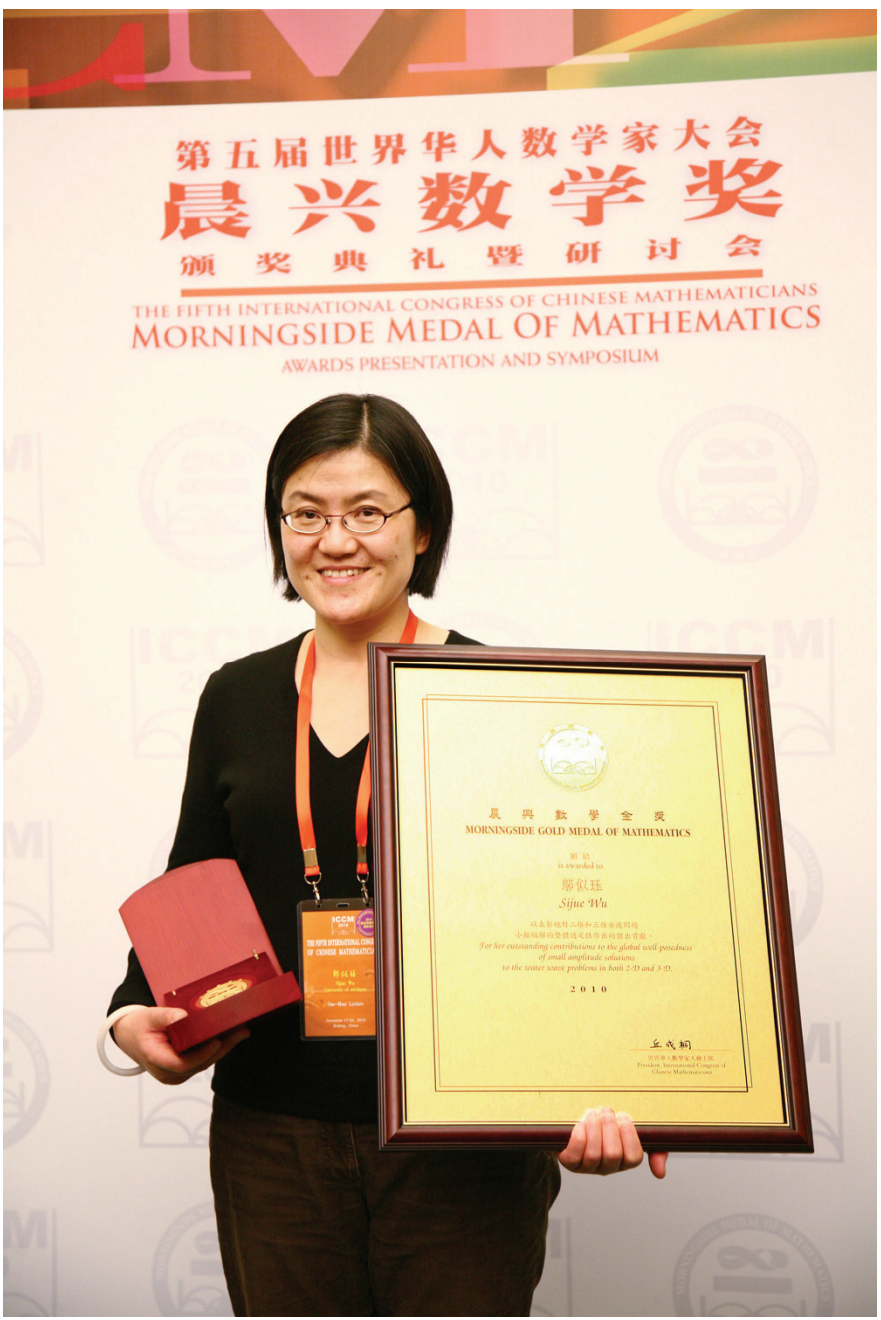

2010 ICCM Gold Medalist, Sijue Wu

Mathematics at the University of Michigan at Ann Arbor. She received her B.S. and M.S. from Peking University and her Ph.D. from Yale University. Prior to joining the University of Michigan, Professor Wu held appointments at the Courant Institute, the Institute for Advanced Study at Princeton, Northwestern University, the University of Iowa and the University of Maryland at College Park. In 2001 Professor Wu was awarded a Morningside Silver Medal of Mathematics and a Ruth Lyttle Satter Prize by the American Mathematics Society. The prize is awarded every two years to recognize an outstanding contribution to mathematics research by a woman in the previous five years. Other academic honors include a Radcliffe Institute for Advanced Study Fellowship at Harvard University, for 2002-2003, and a U.S. National Science Foundation Grant, for 2004-2009.

\section{Morningside Gold Medal of Applied Mathematics (Beijing)}

\section{Jun S. Liu}

Professor Liu is awarded the 2010 Morningside Gold Medal of Applied Mathematics for his fundamental contributions to Monte Carlo inference. He has clarified the properties of Rao-Blackwellization and the collapsed Gibbs sampler, and cointroduced sequential importance sampling into general Bayesian computation. By developing the Gibbs motif sampler, his work also has a profound influence on computational biology. Professor Liu is a Professor of Statistics at Harvard University and a Professor of Biostatistics at the Harvard School of Public Health. He received his B.S. in mathematics from Peking University, continued his studies at Rutgers University and received his Ph.D. in statistics from the University of Chicago. Prior to re-joining Harvard, Professor Liu has held appointments at the National Center for Biotechnology Information, Harvard University, the National University of Singapore, the University of California at Los Angeles, Stanford University, and Peking University. His honors include being a Bernoulli Lecturer and an Institute of Mathematics Statistics (IMS) Fellow in 2004, being a IMS Medallion Lecturer and receiving the COPSS president' Award in 2002, the Mitchell Prize in 2000, and a Terman Fellowship, for 1995-1998.

\section{Morningside Silver Medal of Mathematics (Beijing)}

\section{Jungkai Alfred Chen, Meng Chen}

Professor Jungkai Alfred Chen and Professor Meng Chen are jointly awarded the 2010 Morningside Silver Medal of Mathematics for their breakthrough in the explicit birational classification of algebraic threefolds. In particular, they determined the effective optimal bound for the pluri-canonical system to give rise to birational maps for threefolds of general type. This is the best and in fact the only complete result after Bombieri's famous similar work for surfaces. They also successfully applied their theory to the study of Fano threefolds to get optimal boundedness. 
Professor Jungkai Alfred Chen is a professor of Mathematics at Taiwan University. He previously taught at Chung Cheng University in Taiwan. He also serves as a member of the Taida Institute for Mathematical Sciences and the Center for Theoretical Sciences in Taiwan.

Professor Meng Chen is the Chang Jiang Scholars Program Chair Professor at the School of Mathematical Sciences at Fudan University. He received his B.S., M.S. and D.S. degrees from East China Normal University. Prior to
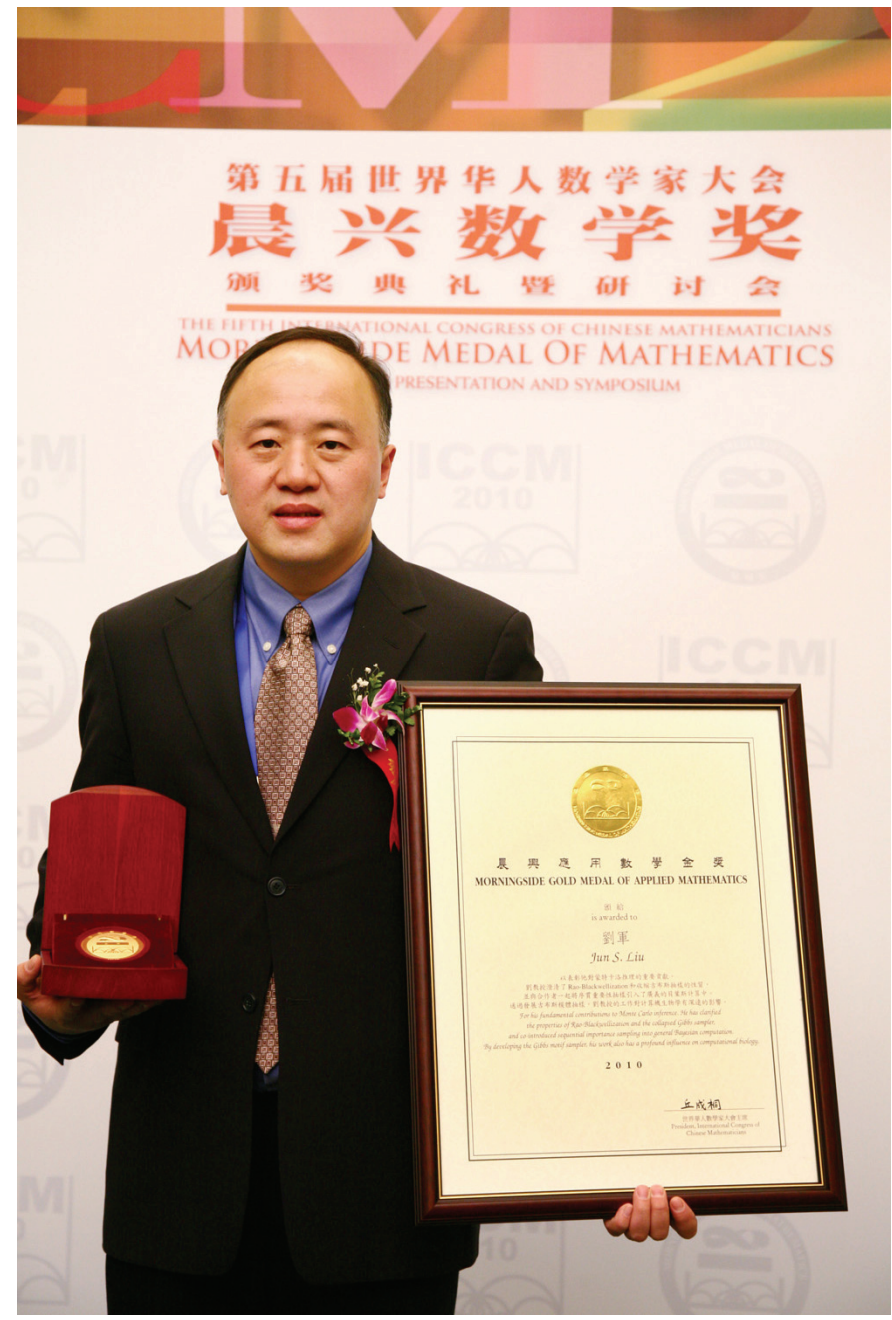

2010 ICCM Applied Mathematics Gold Medalist, Jun Liu

joining the Fudan faculty, Professor Chen taught at Tongji University.

\section{Jixiang $\mathrm{Fu}$}

Professor Fu is awarded the 2010 Morningside Silver Medal of Mathematics for his pioneering work on the existence of Strominger's systems on non-Kähler Calabi-Yau threefolds, and for his proof of the existence of balance metrics on Calabi-Yau threefolds under conifold transition. Professor Fu's works are significant contributions to the study of non-Kähler Calabi-Yau manifolds. Professor $\mathrm{Fu}$ is a professor at the school of Mathematical Sciences at
Fudan University. He was an invited speaker at the 2010 International Congress of Mathematicians in Hyderabad, India.

\section{Juncheng Wei}

Professor Wei is awarded the 2010 Morningside Silver Medal of Mathematics for his achievements in semi-linear elliptic equations. An outstanding one is the counterexample to the de Giorgi conjecture when the space dimension is larger than eight. Another significant one is the existence of multiple peak solutions for a singularly perturbed Neumann Problem. He also obtained the existence of infinitely many solutions to the prescribing scalar curvature equation on the unit sphere. Professor Wei is a professor of Mathematics at The Chinese University of Hong Kong. He obtained his B.S. in mathematics from Wuhan University and his Ph.D. from the University of

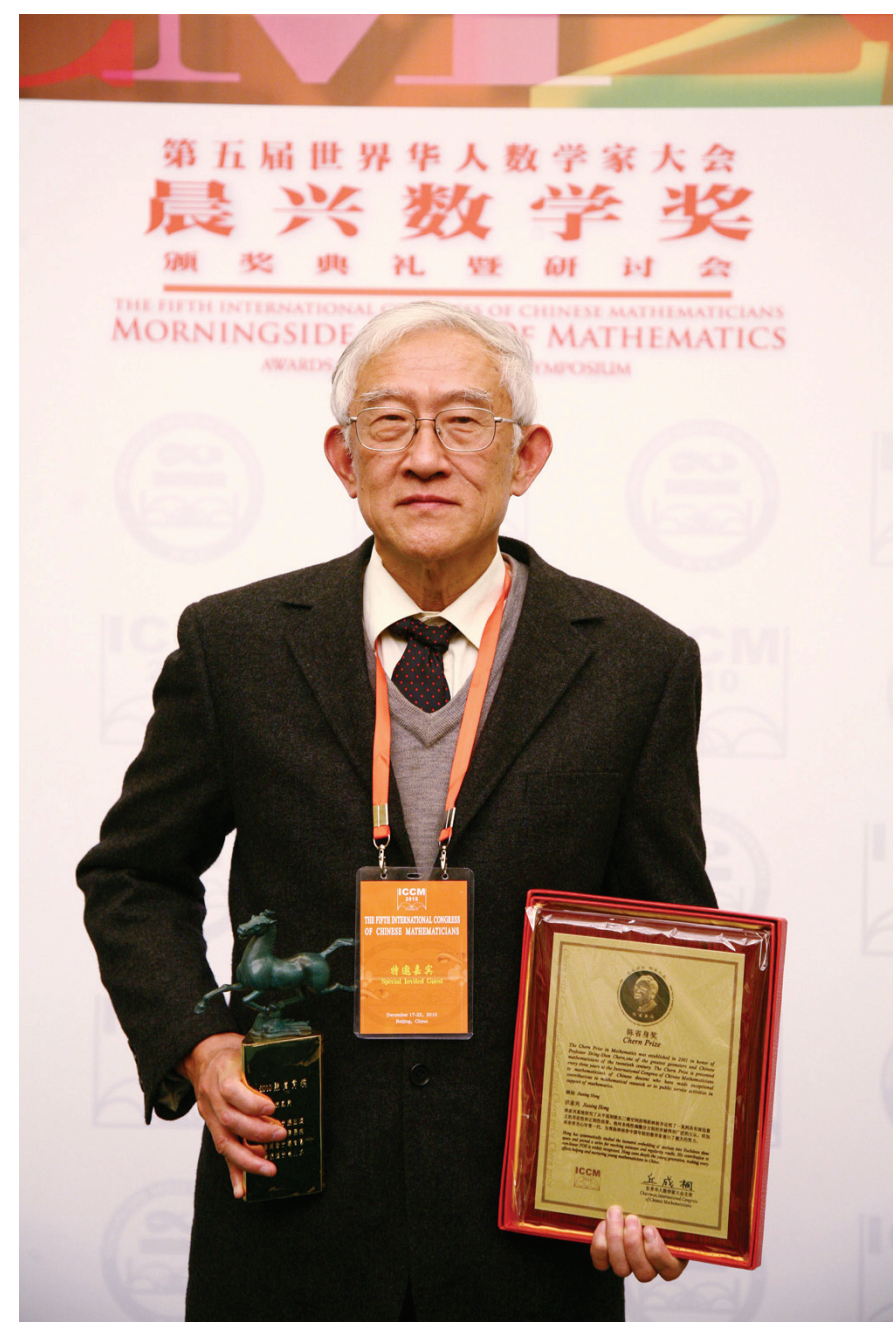

The 2010 ICCM Chern Prize Awardee, Jiaxing Hong

Minnesota. Professor Wei was a 2005 Croucher Senior Research Fellow, and has published more than 120 papers and has spoken at more than 40 international conferences. 


\section{Chern prize}

\section{Jiaxing Hong}

Professor Hong is awarded the 2010 Chern Prize for his systematic study of the isometric embedding of surfaces into Euclidean three-space and his proof of a series of existence and regularity that have far reaching consequences. He is widely recognized for his contribution to non-linear PDE. Professor Hong cares deeply about the young generation, making every effort to help and nur-

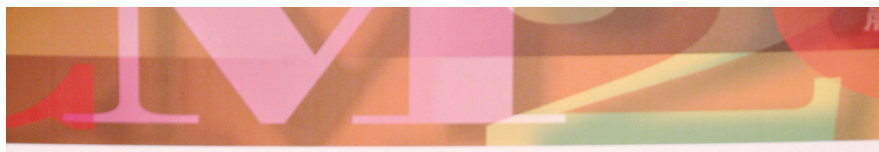

第五届位界华人数学家大会

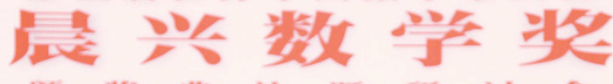

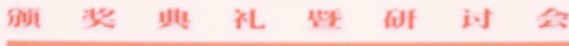

MORNINGSIDE MEDAL OF MATHEMATICS

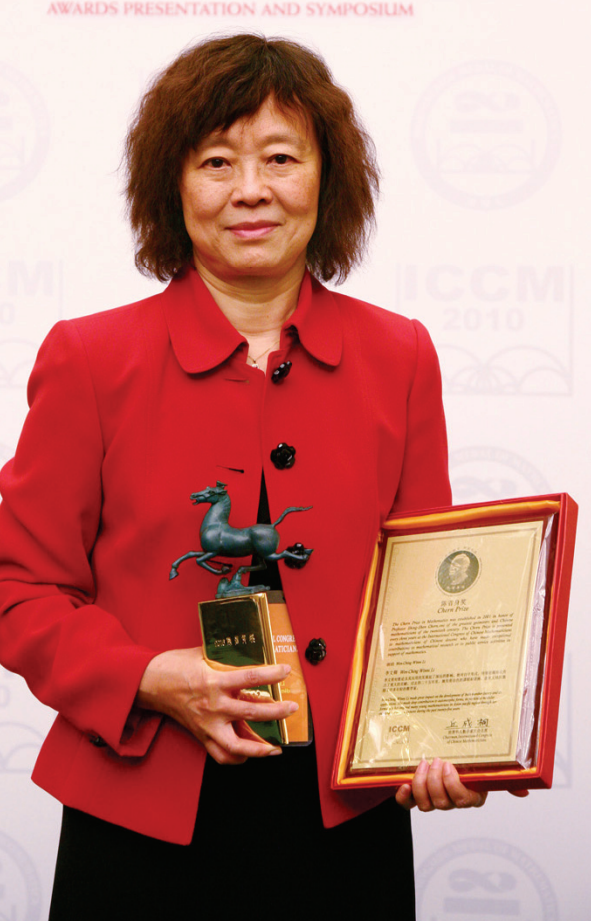

The 2010 ICCM Chern Prize Awardee, Wen-Ching Winnie Li

ture young mathematicians in China. Professor Hong is a professor at the School of Mathematical Sciences at Fudan University and the Superintendent of the University's Institute of Mathematics. He is also the Director of the Laboratory of Nonlinear Mathematical Models and Methods under the Ministry of Education, and serves as an editor for the Chinese Annals of Mathematics and The Asian Journal of Mathematics. He is a member of the Chinese Academy of Sciences.

\section{Wen-Ching Li}

Professor Wen-Ching $\mathrm{Li}$ is an internationally renowned number theorist. In the last two decades, with her expertise on number theory, she works beautifully into the frontiers of spectral graph theory and coding theory. She has inspired many young mathematicians in Asian Pacific region through her excellent courses and lectures during the past 25 years. Currently she is a professor of Pennsylvania State University and a professor of National Tsinghua University at Hsinchu. She also serves as the director of the National Center for Theoretic Sciences in
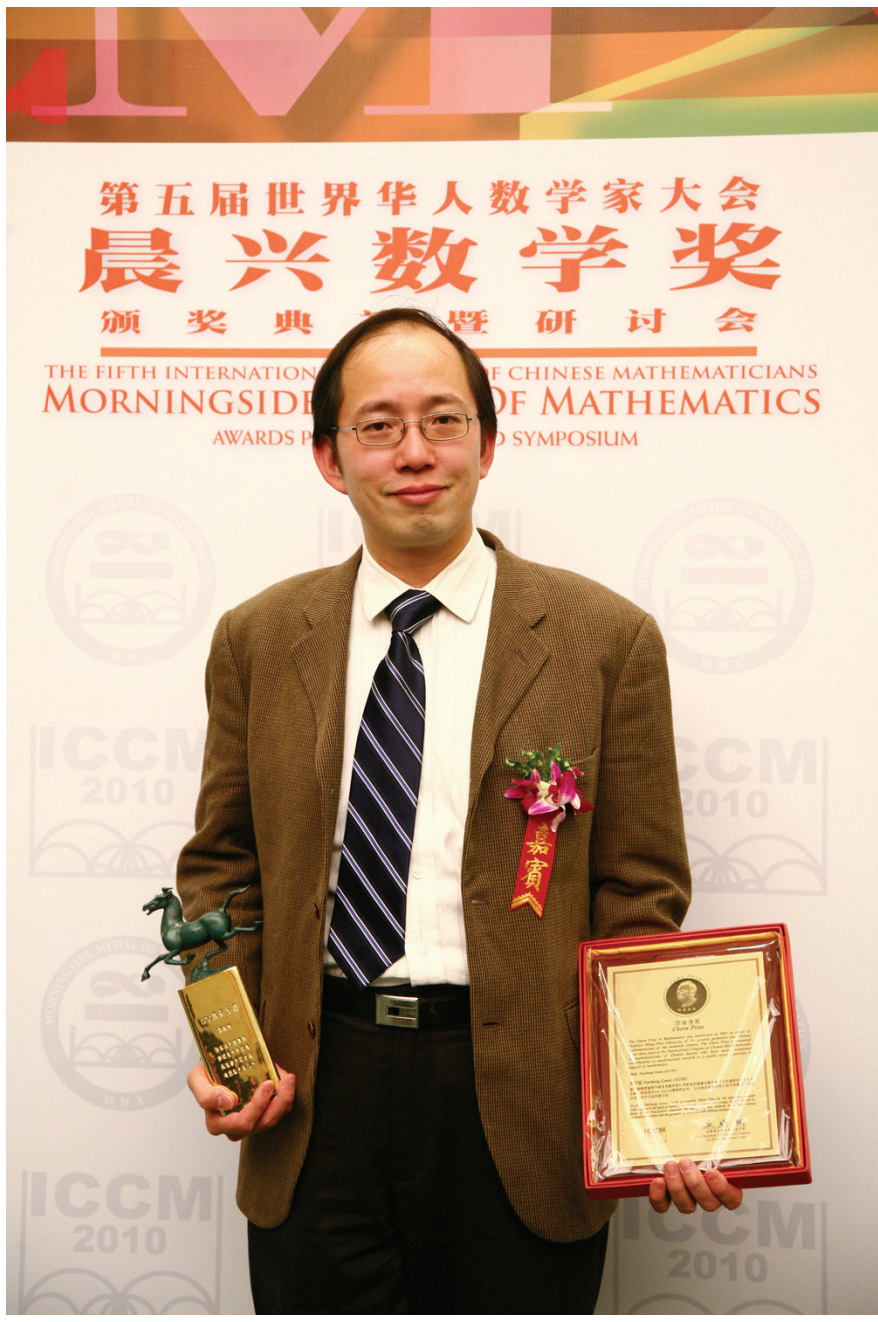

The 2010 ICCM Chern Prize Awardee, Conan Nai-Chung Leung

Taiwan. Together with her predecessors, they promote mathematical researches in Taiwan monumentally.

\section{Conan Nai-Chung Leung}

Professor Leung is awarded the 2010 Chern Prize for his significant research contributions in the study of mirror symmetry and quantum cohomology, in particular for his proof of the Yau-Zaslow conjecture, for introducing new methods in studying various geometric invariants and the geometry of manifolds with different holonomy. Professor Leung has helped organize many international 
mathematics conferences, including the 2007 International Conference on Geometric Analysis held at Taiwan University and the fourth Yamabe Symposium held at the University of Minnesota in 2008. He is an editor of the New York Journal of Mathematics and The Asian Journal of Mathematics. Professor Leung is a professor of Mathematics at The Chinese University of Hong Kong. He received his M.S. in mathematics from the University of California at San Diego, and his Ph.D. in mathematics from the Massachusetts Institute of Technology.

\section{ICCM International Cooperation \\ Award}

\section{Richard M. Schoen}

Professor Schoen is awarded the third ICCM International Cooperation Award for his steadfast contribution to the training of Chinese mathematicians. Professor Schoen has cultivated close relations with Chinese mathematicians throughout his career. Professor Schoen's own doctoral supervisor at Stanford University was Professor Shing-Tung Yau and now, as the Bass Professor of Humanities and Sciences at Stanford University, more than one third of Professor Schoen's 34 Ph.D. students and many of his post-doctoral researchers hail from mainland China, Hong Kong, and Taiwan. Professor Schoen is also recognized for his efforts in continually fostering the Chinese mathematics community, not only in the United States, but also worldwide. This year, Pro-

fessor Schoen joined the Selection Committee of the Morningside Medal of Mathematics. 